\title{
EULER CHARACTERISTICS FOR LINKS OF SCHUBERT CELLS IN THE SPACE OF COMPLETE FLAGS
}

\author{
B. Z. Shapiro And A. D. Vainshtein
}

\section{$\S 1$. INTRODUCTION}

Let $\mathbf{F}_{n}$ be the space of complete flags in $\mathbf{k}^{n}$ (where $\mathbf{k}$ is $\mathbf{R}$ or $\mathbf{C}$ ). With an arbitrary complete flag $f \in \mathbf{F}_{n}$ we associate the standard Schubert cell decomposition $\operatorname{Sch}_{f}$ of the space $\mathbf{F}_{n}$ whose cells are enumerated by elements from $\mathbf{S}_{n}$ while the dimension over $\mathbf{k}$ of such a cell equals the number of inversions in the corresponding permutation (see for example $[\mathrm{FF}] \S 5.4$ ).

Definition. The train $\mathbf{T n}_{f}$ of the flag $f \in \mathbf{F}_{n}$ is the union of all cells of $\operatorname{Sch}_{f}$ of positive codimension.

Let $c_{\sigma}$ be the cell of the decomposition $\operatorname{Sch}_{f}$ corresponding to the permutation $\sigma$ and $B$ a sufficiently small $n(n-1) / 2$-dimensional (over $\mathbf{k}$ ) ball with the origin at some point of $c_{\sigma}$.

Definition. The manifold $A_{\sigma}=B \backslash \mathbf{T n}_{f}$ is called the link of the cell $c_{\sigma}$. By $\chi_{\sigma}$ we denote the Euler characteristic of $A_{\sigma}$ :

$$
\chi_{\sigma}=\sum_{k}(-1)^{k} \operatorname{dim} H^{k}\left(A_{\sigma}\right) .
$$

In the complex case we introduce also the numbers

$$
\chi_{\sigma}^{p q}=\sum_{k}(-1)^{k} \operatorname{dim} \operatorname{Gr}_{F}^{p} \operatorname{Gr}_{p+q}^{W} H^{k}\left(A_{\sigma}\right)
$$

where $\mathrm{Gr}^{W}$ and $\operatorname{Gr}_{F}$ are the associated graded objects of the weight and the Hodge filtrations, respectively.

In this paper we describe a construction which enables us to reduce the calculation of $\chi_{\sigma}$ and $\chi_{\sigma}^{p q}$ for $\mathbf{F}_{n}$ to similar calculations for $\mathbf{F}_{n-1}$ and give the results of the calculations in low dimensions. We also formulate a relation between $\chi_{\sigma}$ for the real case and $\chi_{\sigma}^{p q}$ for the complex one and establish certain properties of the latter numbers.

\section{$\S 2$. Sylvester manifolds, Flags transversal to A GiVEN PAIR OF FLAGS AND LINKS OF SCHUBERT CELLS}

2.1. Let $M$ be an arbitrary matrix over the ring of polynomials in $d$ variables with the coefficients from the field $\mathbf{k}$.

Definition. The Sylvester polynomial of the matrix $M$ is the product of all its main minors; the Sylvester manifold of the matrix $M$ is the complement in $\mathbf{k}^{d}$ to the set of zeros of the Sylvester polynomial of $M$. 
Let $\sigma$ be an arbitrary permutation from $\mathbf{S}_{n}$. Assign to permutation $\sigma$ a following matrix $M_{\sigma}$ over the ring of polynomials in $n(n-1) / 2$ variables: take the upper triangular matrix with unit diagonal elements and independent variables $x_{i j}$ above the diagonal and permutate its columns with the help of $\sigma$.

EXAMPLE. Let $\sigma=(2,3,1,4)$, then

$$
M_{\sigma}=\left(\begin{array}{cccc}
x_{13} & 1 & x_{12} & x_{14} \\
x_{23} & 0 & 1 & x_{24} \\
1 & 0 & 0 & x_{34} \\
0 & 0 & 0 & 1
\end{array}\right)
$$

The Sylvester polynomial of this matrix equals $x_{14} x_{34}\left(x_{12} x_{24}-x_{14}\right)$.

2.2. Let $e_{1}, \ldots, e_{n}$ be an arbitrary basis in $\mathbf{k}^{n}$.

Definition. A complete flag is called coordinate if all its subspaces are spanned by the vectors of the basis and standard coordinate if each its $i$ dimensional subspace is spanned by $e_{1}, \ldots, e_{i}$.

Coordinate flags are enumerated by permutations and each Schubert cell of the Schubert decomposition associated with a standard coordinate flag contains the unique coordinate flag while the permutations corresponding to these flag and cell coincide.

Given a permutation $\sigma=\left(i_{1}, i_{2}, \ldots, i_{n}\right)$, we call the permutation $\bar{\sigma}=\left(i_{n}, \ldots\right.$, $\left.i_{2}, i_{1}\right)$ transversal to $\sigma$. The coordinate flag $f_{\bar{\sigma}}$ corresponding to the permutation $\bar{\sigma}$ is the unique coordinate flag transversal to $f_{\sigma}$.

Let $\left(f_{1}, f_{2}\right)$ be an arbitrary pair of flags in $\mathbf{F}_{n}$. Evidently, there exists a basis in $\mathbf{k}^{n}$ for which $f_{1}$ is the standard flag and $f_{2}$ is a coordinate flag. Each such basis can be obtained from another one via the multiplication by a nondegenerate lower triangular matrix. Thus a permutation $\sigma \in \mathbf{S}_{n}$ is assigned to each pair of flags from $\mathbf{F}_{n}$. The manifold of all flags transversal to a given pair of flags will be denoted by $V_{\sigma}$.

In what follows we shall often use the following fibration $\mathbf{G L}_{n}(\mathbf{k}) \rightarrow \mathbf{F}_{n}$. Fix some basis in $\mathbf{k}^{n}$ thus identifying $\mathbf{G L}_{n}(\mathbf{k})$ with the set of the nondegenerate $n \times$ $n$-matrices and map each matrix onto the flag whose $i$-dimensional subspace is spanned by the first $i$ rows of the matrix.

2.3. Lemma. The manifolds $A_{\sigma}$ and $V_{\bar{\sigma}}$ are diffeomorphic to the Sylvester manifold of the matrix $M_{\sigma}$.

Proof. At first we prove the statement about $V_{\bar{\sigma}}$. The matrix $M_{\sigma}$ defines the mapping of $\mathbf{k}^{n(n-1) / 2}$ to $\mathbf{G L}_{n}(\mathbf{k})$. Let us ascertain now that the set of all flags transversal to $f_{\bar{\sigma}}$ can be identified with the image of this mapping. Suppose $g$ is such a flag and $\bar{\sigma}=\left(i_{1}, \ldots, i_{n}\right)$. Since the line of the flag $g$ is transversal to the linear subspace spanned by $e_{i_{1}}, \ldots, e_{i_{n-1}}$, it contains a unique vector whose projection along $\left\{e_{i_{1}}, \ldots, e_{i_{n-1}}\right\}$ coincides with the basis vector $e_{i_{n}}$; we place the coordinates of this vector in the first row of our matrix. Since the 2-plane of the flag $g$ is transversal to the subspace spanned by $e_{i_{1}}, \ldots, e_{i_{n-2}}$, it contains a unique vector whose projection along $\left\{e_{i_{1}}, \ldots, e_{i_{n-2}}\right\}$ coincides with the basis vector $e_{i_{n-1}}$; we place the coordinates of this vector in the second row of the matrix, etc. At the end of this process we obtain a matrix belonging, obviously, to the image of the mapping determined by $M_{\sigma}$. Consider now the condition that the flag $g$ represented by this matrix is transversal to the standard coordinate flag (represented by the 
unit matrix). The transversality of the $(n-i)$-dimensional subspace of the standard flag and of the $i$-dimensional subspace of $g$ is equivalent to the nondegeneracy of the matrix constituted of the first $n-i$ rows of the unitary matrix and the first $i$ rows of the matrix representing $g$, i.e. to the nonvanishing of the $i$-th main minor of the latter.

Now we prove the statement concerning $A_{\sigma}$. Consider the set of all flags belonging to the $n(n-1) / 2$-dimensional ball with the origin at $f_{\sigma}$. If the radius of this ball is sufficiently small then all these flags are transversal to $f_{\bar{\sigma}}$. Therefore by previous arguments the set of these flags is identified with the image of a small ball under the mapping to $\mathbf{G L}_{n}(\mathbf{k})$ defined by the matrix $M_{\sigma}$. Just as before, the fact that a flag does not belong to the train of the initial (standard coordinate) flag is equivalent to the nonvanishing of the Sylvester polynomial of the matrix $M_{\sigma}$.

\section{§3. Stratification of the Manifold $A_{\sigma}$}

3.1. The $n$-dimensional $\mathbf{k}$-torus $T_{n}=(\mathbf{k} \backslash 0)^{n}$ acts on the manifold $A_{\sigma}$. In the coordinate representation given in $\S 2$ this action can be described as expansions and contractions of basis vectors. The orbits of this action have the following convenient description.

Consider the mapping sending each flag from $A_{\sigma}$ to its line. This line determines an $n$-dimensional vector of 0 's and 1's whose $i$-th coordinate equals 0 if the line belongs $t$ the subspace spanned by $e_{\sigma(1)}, \ldots, e_{\sigma(i-1)}, e_{\sigma(i+1)}, \ldots, e_{\sigma(n)}$ and 1 otherwise. The transversality of all flags from $A_{\sigma}$ to the given pair of flags (see Lemma 2.3) implies that two coordinates (coinciding if the hyperplanes of these two flags coincide) of this vector must equal 1 . Clear that two flags determine the same 0-1-vector if and only if they both belong to the same orbit. Therefore the orbits are enumerated by 0-1-vectors having 1's at two prescribed places (possibly coinciding):

$$
A_{\sigma}=\bigcup_{w \in W_{\sigma}} O_{w \sigma}
$$

where

$$
W_{\sigma}=\left\{w=\left(w_{1}, \ldots, w_{n}\right) \in\{0,1\}^{n}: w_{1}=w_{\sigma^{-1}(n)}=1\right\},
$$

$O_{w \sigma}$ is the orbit in $A_{\sigma}$ corresponding to the vector $w$.

We shall prove now that $O_{w \sigma}$ is diffeomorphic to the product of several copies of $\mathbf{k}^{*}$ by the manifold $A_{\pi}$ for a certain permutation $\pi \in \mathbf{S}_{n-1}$.

Let $\sigma \in \mathbf{S}_{n}, w \in W_{\sigma}$. To each pair $(\sigma, w)$ we assign a permutation $\pi(\sigma, w) \in \mathbf{S}_{n-1}$ in the following way.

Given an arbitrary sequence $I=\left\{i_{1}, \ldots, i_{k}\right\}$ denote by $R(I)$ the sequence obtained by the following process: put

$$
r_{1}=i_{1}, \quad r_{l}=\max \left\{r_{l-1}, i_{l}\right\}, \quad 1<l \leq k,
$$

and delete from each group of consecutive equal elements of the sequence $\left\{r_{1}, \ldots\right.$, $\left.r_{k}\right\}$ all elements except the first one. Now let $I(w)$ be the ordered sequence of the numbers $i$ such that $w_{i}=1$. Put $J_{\sigma}(w)=\sigma^{-1} R(\sigma I(w))$; then a relation $1=j_{1}<j_{2}<\cdots<j_{m}=\sigma^{-1}(n)$ is obviously valid, $m$ being the number of elements in $J_{\sigma}(w)$. Define $\pi(\sigma, w)$ by the formulas

$$
\begin{aligned}
& \pi(\sigma, w)(i)=\sigma(i+1) \quad \text { if } i \neq j_{l}-1, \quad 2 \leq l \leq m, \\
& \pi(\sigma, w)\left(j_{l}-1\right)=\sigma\left(j_{l-1}\right), \quad 2 \leq l \leq m \text {. }
\end{aligned}
$$


3.2. Lemma. The manifold $O_{w \sigma}$ is diffeomorphic to the direct product of $A_{\pi(\sigma, w)}$ by $\left(\mathbf{k}^{*}\right)^{n(w)-1}$, where $n(w)$ is the number of nonzero entries in $w$.

Proof. Note that $O_{w \sigma} \cong A_{w \sigma} \times\left(\mathbf{k}^{*}\right)^{n(w)-1}$, where $A_{w \sigma}$ is the Sylvester manifold of the matrix $M_{w \sigma}$ obtained from $M_{\sigma}$ via replacing its first row by the vector $w \sigma=\left(w_{\sigma^{-1}(1)}, \ldots, w_{\sigma^{-1}(n)}\right)$. Let us prove that the Sylvester manifolds of $M_{w \sigma}$ and of $M_{\pi(\sigma, w)}$ are diffeomorphic. Indeed, put $\left\{r_{1}, \ldots, r_{m}\right\}=R(\sigma I(w)), r_{0}=0$ and define the matrix $L$ by the relations

$$
\begin{aligned}
& l_{i i}=(-1)^{w_{\sigma(i)}}, \quad 1 \leq i \leq n ; \\
& l_{i j}= \begin{cases}1 & \text { if } w_{\sigma(j)}=1, r_{k-1} \leq j \leq r_{k}, k \in\{1, \ldots, m\} ; \\
0 & \text { otherwise }\end{cases}
\end{aligned}
$$

Since $L$ is a nondegenerate lower triangular matrix, the Sylvester polynomials for $M_{w \sigma}$ and $M_{w \sigma} L$ coincide up to the factor \pm 1 . The first row of $M_{w \sigma} L$ contains the unique unity: in the last column. Hence, the Sylvester polynomial for this matrix is equal to that for its submatrix obtained by deleting of the first row and of the last column; however, by linear changes of variables the latter can be reduced to $M_{\pi(\sigma, w)}$, q.e.d.

3.3. Call vectors $w^{1}, w^{2} \in W_{\sigma}$ equivalent $\left(w^{1} \sim w^{2}\right)$ if $\pi\left(\sigma, w^{1}\right)=\pi\left(\sigma, w^{2}\right)$. Relations (2) imply

$$
w^{1} \sim w^{2} \quad \text { iff } \quad J_{\sigma}\left(w^{1}\right)=J_{\sigma}\left(w^{2}\right) .
$$

Denote by $\tilde{W}_{\sigma}$ the quotient set $W_{\sigma} / \sim$ and by $\tilde{W}_{\sigma}(w)$ the equivalence class containing $w$.

The set $\{1, \ldots, n\}$ can be decomposed in the disjoint union of the three subsets $N_{0}, N_{1}$ and $N_{01}$. Here $N_{0}$ contains all $j$ such that $w_{j}^{\prime}=0$ for all $w^{\prime} \in \tilde{W}_{\sigma}(w), N_{1}$ contains all $j$ such that $w_{j}^{\prime}=1$ for all $w^{\prime} \in \tilde{W}_{\sigma}(w), N_{01}$ contains all other $j$ 's. The definition of $J_{\sigma}$ implies that

$$
\begin{array}{lll}
i \in N_{1} & \text { iff } & i \in J_{\sigma}(w) ; \\
i \in N_{0} & \text { iff } & \exists l: j_{l}<i<j_{l+1}, \sigma(i)>\sigma\left(j_{l+1}\right) .
\end{array}
$$

Define vectors $\underline{w}$ and $\bar{w}$ by the relations

$$
\underline{w}_{i}=\left\{\begin{array}{ll}
1 & \text { if } i \in N_{1}, \\
0 & \text { otherwise, }
\end{array} \quad \bar{w}_{i}=\left\{\begin{array}{ll}
0 & \text { if } \quad i \in N_{0}, \\
1 & \text { otherwise, }
\end{array} \quad 1 \leq i \leq n .\right.\right.
$$

It is easy to see that $\tilde{W}_{\sigma}(w)=\left\{w^{\prime}: \underline{w}_{i} \leq w_{i}^{\prime} \leq \bar{w}_{i}, i=1, \ldots, n\right\}$.

For an arbitrary permutation $\tau$ denote by $D_{\tau}$ the set of all pairs $(i, k)$ such that $i<k$ and $\tau(i)<\tau(k)$ and let $d_{\tau}$ be the cardinality of $D_{\tau}$.

3.4. Lemma. For arbitrary $\sigma \in \mathbf{S}_{n}, w \in W_{\sigma}$

$$
d_{\sigma}-d_{\pi(\sigma, w)}=n(\underline{w})-1+2(n-n(\bar{w})) .
$$

Proof. Let us define a mapping $\pi^{*}: D_{\pi} \rightarrow D_{\sigma}$ (since $\sigma$ and $w$ are fixed, we can and will omit dependence of $\pi$ and of $\pi^{*}$ on these parameters). To do this, decompose each of $D_{\sigma}$ and $D_{\pi}$ in 6 subsets. 
Let $(i, k) \in D_{\sigma}$. Put

$$
\begin{array}{llll}
(i, k) \in D_{\sigma}^{1} & \text { iff } & i \notin N_{1}, k \notin N_{1}, \\
(i, k) \in D_{\sigma}^{2} & \text { iff } & i \in N_{1}, k \in N_{01}, \\
(i, k) \in D_{\sigma}^{3} & \text { iff } & i \in N_{01}, k \in N_{1}, \\
(i, k) \in D_{\sigma}^{4} & \text { iff } & i \in N_{1}, k \in N_{0}, \\
(i, k) \in D_{\sigma}^{5} & \text { iff } & i \in N_{0}, k \in N_{1}, \\
(i, k) \in D_{\sigma}^{6} & \text { iff } & i \in N_{1}, k \in N_{1} .
\end{array}
$$

The corresponding partition of $D_{\pi}$ is defined in the following way: let $(i, k) \in D_{\pi}$, then

$$
(i, k) \in D_{\pi}^{l} \quad \text { iff } \quad(i+1, k+1) \in D_{\sigma}^{l}, \quad 1 \leq l \leq 6 .
$$

For $(i, k) \in D_{\pi}^{1}$ put $\pi^{*}(i, k)=(i+1, k+1)$. We have $i+1<k+1$ while $\sigma(i+1)=\pi(i)<\pi(k)=\sigma(k+1)$ by $(2)$, hence $(i+1, k+1) \in D_{\sigma}^{1}$. Evidently, $\pi^{*}$ defines a bijection of $D_{\pi}^{1}$ and $D_{\sigma}^{1}$.

For $(i, k) \in D_{\pi}^{2}$ we have $i+1=j_{l}, l>1$ (recall that $j_{l}$ is the $l$ 'th element in $\left.J_{\sigma}(w)\right)$. Put $\pi^{*}(i, k)=\left(j_{l-1}, k+1\right)$. Then $j_{l-1}<j_{l}=i+1<k+1$ and by $(2)$ $\sigma\left(j_{l-1}\right)=\pi\left(j_{l}-1\right)<\pi(k)=\sigma(k+1)$, hence $\left(j_{l-1}, k+1\right) \in D_{\sigma}^{2}$. Since $(s, t) \in D_{\sigma}^{2}$ implies the existence of $s^{\prime} \in N_{1}$ such that $s<s^{\prime}<t$, we see that $\pi^{*}$ defines a bijection of $D_{\pi}^{2}$ and $D_{\sigma}^{2}$.

For $(i, k) \in D_{\pi}^{3}$ we have $k+1=j_{l}, l>1$. Put $\pi^{*}(i, k)=\left(i+1, j_{l}\right)$. We have $i+1<j_{l}$ while by $(2) \sigma(i+1)=\pi(i)<\pi\left(j_{l}-1\right)=\sigma\left(j_{l-1}\right)<\sigma\left(j_{l}\right)$, hence $\left(i+1, j_{l}\right) \in D_{\sigma}^{3}$. Evidently, $\pi^{*}$ defines a bijection of $D_{\pi}^{3}$ and $D_{\sigma}^{3}$.

For $(i, k) \in D_{\pi}^{4}$ we have $i+1=j_{l}, l>1$. Put $\pi^{*}(i, k)=\left(j_{l-1}, k+1\right)$. As in the case of $D_{\pi}^{2}$ we obtain $\left(j_{l-1}, k+1\right) \in D_{\sigma}^{4}$. Evidently, $\pi^{*}$ is injective on $D_{\pi}^{4}$. Let $(s, t) \in D_{\sigma}^{4}$; from the definition of $J_{\sigma}(w)$ it follows that $(s, t) \in \pi^{*}\left(D_{\pi}^{4}\right)$ iff there exists $s^{\prime}$ such that $s<s^{\prime}<t$. Since for each $t \in N_{0}$ there exists a unique $s \in N_{1}$ such that the pair $(s, t)$ does not possess the above property, we see that the number of elements in $D_{\sigma}^{4}$ exceeds that in $D_{\pi}^{4}$ by card $N_{0}=n-n(\bar{w})$.

For $(i, k) \in D_{\pi}^{5}$ we have $k+1=j_{l}, l>1$. Put $\pi^{*}(i, k)=\left(i+1, j_{l-1}\right)$. By $(2)$ $\sigma(i+1)=\pi(i)<\pi\left(j_{l}-1\right)=\sigma\left(j_{l-1}\right)$. Let us prove that $i+1<j_{l-1}$. Indeed, by the definition of $N_{0}$ the opposite inequality would imply $\sigma\left(J_{l-1}\right)<\sigma(i+1)$, i.e. $\pi(k)<\pi(i)$ which contradicts $(i, k) \in D_{\pi}^{5}$. Therefore $\left(i+1, j_{l-1}\right) \in D_{\sigma}^{5}$. Evidently, $\pi^{*}$ is injective on $D_{\pi}^{5}$. Let $(s, t) \in D_{\sigma}^{5}$; from the definition of $J_{\sigma}(w)$ follows that $(s, t) \in \pi^{*}\left(D_{\pi}^{5}\right)$ iff $t \neq j_{m}$ (as before, $m$ denotes the cardinality of $J_{\sigma}(w)$ ). Since for each $s \in N_{0} D_{\sigma}^{5}$ contains the unique pair $\left(s, j_{m}\right)$, we see that the number of elements in $D_{\sigma}^{5}$ exceeds that in $D_{\pi}^{5}$ by card $N_{0}=n-n(\bar{w})$.

For $(i, k) \in D_{\pi}^{6}$ we have $i+1=j_{l}, k+1=j_{r}, 1<l<r$. Put $\pi^{*}(i, k)=$ $\left(j_{l-1}, j_{r-1}\right)$. We have $j_{l-1}<j_{r-1}$ while by $(2) \sigma\left(j_{l-1}\right)=\pi\left(j_{l}-1\right)<\pi\left(j_{r}-1\right)=$ $\sigma\left(j_{r-1}\right)$, hence $\left(j_{l-1}, j_{r-1}\right) \in D_{\sigma}^{6}$. Evidently, $\pi^{*}$ is injective on $D_{\pi}^{6}$. Let $(s, t) \in D_{\sigma}^{6}$; from the definition of $J_{\sigma}(w)$ follows that $(s, t) \in \pi^{*}\left(D_{\pi}^{6}\right)$ iff $t \neq j_{m}$. Since $D_{\sigma}^{6}$ contains the unique pair $\left(s, j_{m}\right)$ for each $s \in N_{1}, s \neq j_{m}$, we see that the number of elements in $D_{\sigma}^{6}$ exceeds that in $D_{\pi}^{6}$ by card $n_{1}=n(\underline{w})-1$.

Therefore

$$
d_{\sigma}-d_{\pi}=\sum_{l=1}^{6}\left(\operatorname{card} D_{\sigma}^{l}-\operatorname{card} D_{\pi}^{l}\right)=n(\underline{w})-1+2(n-n(\bar{w}))
$$

q.e.d. 


\section{$\S 4$. EULER CHARACTERISTICS OF STRATIFIED MANIFOLDS FOR COHOMOLOGY WITH COMPACT SUPPORTS}

4.1. It is known (see e.g. $[\mathrm{M}]$ ) that the theory of cohomology with compact supports is "the theory of a single space". It means that if $Y \subset X$ is a closed subset of a locally compact topological space $X$ then for $U=X \backslash Y$ one has $H_{c}^{i}(X, Y) \equiv H_{c}^{i}(U)$ and the exact sequence of the pair can be written as

$$
\cdots \rightarrow H_{c}^{k}(U) \rightarrow H_{c}^{k}(X) \rightarrow H_{c}^{k}(Y) \rightarrow H_{c}^{k+1}(U) \rightarrow \ldots
$$

thus implying

$$
\chi_{c}(X)=\chi_{c}(U)+\chi_{c}(Y)
$$

The following proposition was mentioned by many authors (see e.g. [G] and especially $[\mathrm{V}])$.

4.2. Lemma. Suppose $X$ is an $n$-dimensional manifold represented as a finite disjoint union of open manifolds $X_{i}: X=\cup_{i} X_{i}$. Then

$$
\chi_{c}(X)=\sum_{i} \chi_{c}\left(X_{i}\right)
$$

Proof. Consider the filtration $X=X^{n} \supset X^{n-1} \supset X^{n-2} \supset \cdots \supset X^{0}=\emptyset$, where $X^{l}$ is the union of all $X_{i}$ 's whose dimension does not exceed $l$ and apply (4) consequently to the pairs $\left(X^{n}, X^{n-1}\right),\left(X^{n-1}, X^{n-2}\right)$ and so on.

It turns out that a similar proposition is true for $\chi_{c}^{p q}$ 's of quasiprojective manifolds. (From now on in this section we shall follow mainly [D].)

4.3. Theorem. Suppose $X$ is a complex quasiprojective manifold represented as a finite disjoint union of quasiprojective manifolds $X_{i}: X=\cup_{i} X_{i}$. Then

$$
\chi_{c}^{p q}(X)=\sum_{i} \chi_{c}^{p q}\left(X_{i}\right)
$$

The following property of the exact sequence (3) for quasiprojective manifolds immediately implies the Theorem.

4.4. Lemma. Let $Y \subset X$ be a closed quasiprojective submanifold of a quasiprojective manifold $X$ and $U=X \backslash Y$. Then the exact sequence (3) is an exact sequence of Hodge structures.

Proof. Choose a compactification $\bar{X} \supset X$ and denote by $X^{\prime}$ the complement to $X$ in $\bar{X}$, by $\bar{Y}$ - the closure of $Y$ in $\bar{X}$ and by $Y^{\prime}$ - the complement to $Y$ in $\bar{Y}$. Then sequence $(3)$ is reduced to

$$
\cdots \rightarrow H^{k}\left(\bar{X}, X^{\prime} \cup \bar{Y}\right) \rightarrow H^{k}\left(\bar{X}, X^{\prime}\right) \rightarrow H^{k}\left(\bar{Y}, Y^{\prime}\right) \rightarrow H^{k+1}\left(\bar{X}, X^{\prime} \cup \bar{Y}\right) \rightarrow \ldots
$$

By the excision isomorphism, the third group is isomorphic to $H^{k}\left(X^{\prime} \cup \bar{Y}, X^{\prime}\right)$, thus (3) is reduced to the exact sequence of the triple $\left(\bar{X}, X^{\prime} \cup \bar{Y}, X^{\prime}\right)$. However, the exact sequence of a triple is an exact sequence of Hodge structures; to prove 
this it is sufficient to check that Hodge structures are respected by the connecting homomorphism. The latter fact is implied by the similar property of the exact sequence of a pair.

4.5. To prove Theorem 4.3 it suffices to consider the case $X=X_{1} \cup X_{2}$. If both $X_{1}$ and $X_{2}$ are closed the Theorem is implied immediately by Lemma 4.4. In general, let $\bar{X}_{1}$ be the closure of $X_{1}$ in $X$ and $C=\bar{X}_{1} \cap X_{2}$. Then $X_{1}$ is open in $\bar{X}_{1}$ (since $X_{1}$ is quasiprojective), $\bar{X}_{1}$ is closed in $X$ and $C$ is closed in $X_{2}$. Applying Lemma 4.4 three times we obtain

$$
\begin{aligned}
\chi_{c}^{p q}(X) & =\chi_{c}^{p q}\left(\bar{X}_{1}\right)+\chi_{c}^{p q}\left(X_{2} \backslash C\right)= \\
& =\chi_{c}^{p q}\left(X_{1}\right)+\chi_{c}^{p q}(C)+\chi_{c}^{p q}\left(X_{2} \backslash C\right)= \\
& =\chi_{c}^{p q}\left(X_{1}\right)+\chi_{c}^{p q}\left(X_{2}\right),
\end{aligned}
$$

which completes the proof.

\section{$\S 5$. Main Results}

5.1. Theorem. In the real case

$$
\chi_{\sigma}=\sum_{w \in W_{\sigma}}(-1)^{n-n(w)} 2^{n(w)-1} \chi_{\pi(\sigma, w)},
$$

where $n(w)$ as before is the number of unit entries in $w$.

Proof. From (1) and Lemma 4.2 it follows that

$$
\chi_{c}\left(A_{\sigma}\right)=\sum_{w \in W_{\sigma}} \chi_{c}\left(O_{w \sigma}\right) .
$$

Lemma 2.3 and the explicit description of the orbits $O_{w \sigma}$ (see section 3.1) imply $\operatorname{dim} O_{w \sigma}=\frac{n(n-1)}{2}-(n-n(w))$. Hence by Lemma 3.2.

$$
\begin{aligned}
\chi_{c}\left(O_{w \sigma}\right) & =\sum_{k}(-1)^{k} h_{c}^{k}\left(O_{w \sigma}\right)=\sum_{k}(-1)^{k} h^{n(n-1) / 2-n+n(w)-k}\left(O_{w \sigma}\right)= \\
& =\sum_{k}(-1)^{k} h^{0}\left(\left(\mathbf{R}^{*}\right)^{n(w)-1}\right) h^{n(n-1) / 2-n+n(w)-k}\left(A_{\pi(\sigma, w)}\right)= \\
& =2^{n(w)-1} \sum_{k}(-1)^{k} h_{c}^{k+1-n(w)}\left(A_{\pi(\sigma, w)}\right)=(-2)^{n(w)-1} \chi_{c}\left(A_{\pi(\sigma, w)}\right)= \\
& =(-1)^{(n-1)(n-2) / 2}(-2)^{n(w)-1} \chi_{\pi(\sigma, w)} .
\end{aligned}
$$

From the above relation and formula (5) follows that

$$
\chi_{\sigma}=(-1)^{n(n-1) / 2} \chi_{c}\left(A_{\sigma}\right)=\sum_{w \in W_{\sigma}}(-1)^{n-n(w)} 2^{n(w)-1} \chi_{\pi(\sigma, w)},
$$

q.e.d.

5.2. To handle the complex case we need the following technical proposition.

Suppose $X$ is an arbitrary complex manifold; denote

$$
\begin{gathered}
P_{X}(t)=\sum_{i} \chi^{i i}(X) t^{i}, \\
h_{k}^{i j}(X)=\operatorname{dim} \operatorname{Gr}_{F}^{i} \operatorname{Gr}_{i+j}^{W} H^{k}(X) .
\end{gathered}
$$


Lemma. Let $X=U \times Y$ and the following assumptions are true:

1) $\chi^{i j}(Y)=0$ for $i \neq j$;

2) $h_{k}^{i j}(U)=0$ for $i \neq j$.

Then

$$
\begin{gathered}
P_{X}(t)=P_{U}(t) P_{Y}(t), \\
\chi^{i j}(X)=0 \quad \text { for } \quad i \neq j .
\end{gathered}
$$

Proof. Evidently, assumption (2) implies that

$$
h_{k}^{i j}(X)=\sum_{p, q, l} h_{l}^{p q}(U) h_{k-l}^{i-p, j-q}(Y)=\sum_{p, l} h_{l}^{p p}(U) h_{k-l}^{i-p, j-p}(Y) .
$$

Hence

$$
\begin{aligned}
\chi^{i j}(X) & =\sum_{k}(-1)^{k} h_{k}^{i j}(X)=\sum_{p, l}(-1)^{l} h_{l}^{p p}(U) \sum_{k}(-1)^{k-l} h_{k-l}^{i-p, j-p}(Y)= \\
& =\sum_{p, l}(-1)^{l} h_{l}^{p p}(U) \chi^{i-p, j-p}(Y)=\sum_{p} \chi^{p p}(U) \chi^{i-p, j-p}(Y) .
\end{aligned}
$$

From this relation we see that (7) follows immediately from assumption (1). Now,

$$
\begin{aligned}
P_{X}(t) & =\sum_{i} \sum_{p} \chi^{p p}(U) \chi^{i-p, i-p}(Y) t^{i}=\left(\sum_{p} \chi^{p p}(U) t^{p}\right)\left(\sum_{j} \chi^{j j}(Y) t^{j}\right)= \\
& =P_{U}(t) P_{Y}(t),
\end{aligned}
$$

which coincides with (6).

5.3. Theorem. Put $P_{\sigma}(t) \equiv P_{A_{\sigma}}(t)$, then

$$
P_{\sigma}(t)=\sum_{w \in W_{\sigma}} t^{n-n(w)}(1-t)^{n(w)-1} P_{\pi(\sigma, w)}(t),
$$

$$
\chi_{\sigma}^{i j}=0 \quad \text { for } \quad i \neq j,
$$

where $n(w)$ is the same that in Theorem 5.1.

Proof. Denote $P_{w \sigma}(t) \equiv P_{O_{w \sigma}}(t)$. Lemmas 3.2 and 5.2 imply

$$
P_{w \sigma}(t)=P_{\left(\mathbf{C}^{*}\right)^{n(w)-1}} P_{\pi(\sigma, w)}(t)=(1-t)^{n(w)-1} P_{\pi(\sigma, w)}(t) .
$$

For an arbitrary complex manifold $X$ define a polynomial

$$
P_{X}^{c}(t)=\sum_{i} t^{i} \sum_{k} \operatorname{dim} \operatorname{Gr}_{F}^{i} \operatorname{Gr}_{2 i}^{W} H_{c}^{k}(X)
$$

and extend on this the abbreviated notions $P_{\sigma}^{c}$ and $P_{w \sigma}^{c}$. Evidently,

$$
P_{X}^{c}(t)=t^{d} P_{X}(1 / t)
$$

where $d$ is the complex dimension of $X$. According to Theorem 4.3 relation (1) implies

$$
P_{\sigma}^{c}(t)=\sum_{w \in W_{\sigma}} P_{w \sigma}^{c}(t) .
$$

Together with (11) this implies

$$
t^{n(n-1) / 2} P_{\sigma}(1 / t)=\sum_{w \in W_{\sigma}} t^{n(n-1) / 2-n+n(w)} P_{w \sigma}(1 / t) .
$$

Introducing (10) in the above relation and redenoting $\frac{1}{t}$ by $t$ we obtain (8). 
5.4. Corollary. In the complex case $\chi_{\sigma}=0$.

Proof. Evidently, $\chi_{\sigma}=\sum_{i, j} \chi_{\sigma}^{i j}$. Together with (8), (9) this implies $\chi_{\sigma}=\sum_{i} \chi_{\sigma}^{i i}=$ $P_{\sigma}(1)=0$.

5.5. A connection between $\chi_{\sigma}$ for the real case (ad hoc denote it by $\chi_{\sigma}^{\mathbf{R}}$ ) and $\chi_{\sigma}^{i i}$ is given by the following proposition.

Corollary. $\quad \chi_{\sigma}^{\mathbf{R}}=\sum_{i}\left|\chi_{\sigma}^{i i}\right|$.

Proof. Follows immediately from Theorem 5.1, formula (8) and the relation $\sum_{i}\left|\chi_{\sigma}^{i i}\right|=$ $P_{\sigma}(-1)$.

5.6. Theorem. For any $\sigma \in W_{\sigma}$

$$
\begin{gathered}
\operatorname{deg} P_{\sigma}=d_{\sigma}, \quad \chi_{\sigma}^{d_{\sigma} d_{\sigma}}=(-1)^{d_{\sigma}}, \\
\chi_{\sigma}^{i i}=(-1)^{d_{\sigma}} \chi_{\sigma}^{d_{\sigma}-i, d_{\sigma}-i}, \quad 0 \leq i \leq d_{\sigma} .
\end{gathered}
$$

Proof. For an arbitrary $\tilde{w} \in \tilde{W}_{\sigma}$ put

$$
Q_{\tilde{w}}(t)=\sum_{w \in \tilde{w}} t^{n-n(w)}(1-t)^{n(w)-1} P_{\pi(\sigma, w)}(t) .
$$

Then (8) can be rewritten as

$$
P_{\sigma}(t)=\sum_{\tilde{w} \in \tilde{W}_{\sigma}} Q_{\tilde{w}}(t)
$$

while

$$
\begin{aligned}
Q_{\tilde{w}}(t) & =\sum_{i=0}^{n(\bar{w})-n(\underline{w})}\left(\begin{array}{c}
n(\bar{w})-n(\underline{w}) \\
i
\end{array}\right) t^{n-n(\bar{w})+i}(1-t)^{n(\bar{w})-i-1} P_{\pi(\sigma, w)}(t)= \\
& =t^{n-n(\bar{w})}(1-t)^{n(\underline{w})-1} P_{\pi(\sigma, w)}(t)
\end{aligned}
$$

with $w$ satisfying $\tilde{W}_{\sigma}(w)=\tilde{w}$.

Suppose that (12) is already proved for permutations from $\mathbf{S}_{n-1}$ (the basis of the induction is trivial). Then

$$
\operatorname{deg} Q_{\tilde{w}}=n-n(\bar{w})+n(\underline{w})-1+d_{\pi(\sigma, w)} .
$$

By Lemma 3.4 this relation implies $\operatorname{deg} Q_{\tilde{w}}=d_{\sigma}-n+n(\bar{w})$. This means that only one polynomial in the right hand side of (14) has degree $d_{\sigma}$ (the corresponding $w$ consists of all 1's), while the degrees of all other polynomials are strictly less then $d_{\sigma}$. Together with (15) this implies (12).

Now note that by (12) relation (13) is equivalent to

$$
P_{\sigma}(t)=(-t)^{d_{\sigma}} P_{\sigma}(1 / t) .
$$

Suppose that this relation is already proved for permutations from $\mathbf{S}_{n-1}$. From (15) and Lemma 3.4 we see that

$$
Q_{\tilde{w}}(1 / t)=(-t)^{-d_{\sigma}} Q_{\tilde{w}}(t)
$$

Introducing this into (14) we obtain the required relation. 


\section{$\S 6$. Calculations for low Dimensions}

Theorems 5.1 and 5.3 enable us to calculate $\chi_{\sigma}$ and $P_{\sigma}$ for any $\mathbf{S}_{n}$ consecutively by $n$. The results for $n=1,2,3,4$ are displayed below.

\begin{tabular}{|c|c|c|c|}
\hline$n$ & $\sigma$ & $\chi_{\sigma}$ & $P_{\sigma}$ \\
\hline 1 & 1 & 1 & 1 \\
\hline \multirow{2}{*}{2} & $(1,2)$ & 2 & $1-t$ \\
\hline & $(2,1)$ & 1 & 1 \\
\hline \multirow{4}{*}{3} & $(1,2,3)$ & 6 & $1-2 t+2 t^{2}-t^{3}$ \\
\hline & $(1,3,2),(2,1,3)$ & 4 & $1-2 t+t^{2}$ \\
\hline & $(2,3,1),(3,1,2)$ & 2 & $1-t$ \\
\hline & $(3,2,1)$ & 1 & 1 \\
\hline \multirow{15}{*}{4} & $(1,2,3,4)$ & 20 & $1-3 t+4 t^{2}-4 t^{3}+4 t^{4}-3 t^{5}+t^{6}$ \\
\hline & $(1,3,2,4)$ & 18 & $1-3 t+5 t^{2}-5 t^{3}+3 t^{4}-t^{5}$ \\
\hline & $(1,2,4,3),(2,1,3,4)$ & 16 & $1-3 t+4 t^{2}-4 t^{3}+3 t^{4}-t^{5}$ \\
\hline & $(2,1,4,3),(2,3,1,4))$, & & \\
\hline & $(3,1,2,4),(1,3,4,2)\}$, & 12 & $1-3 t+4 t^{2}-3 t^{3}+t^{4}$ \\
\hline & $(1,4,2,3)$ & & \\
\hline & $(3,2,1,4),(1,4,3,2)$, & 8 & $1-3 t+3 t^{2}-t^{3}$ \\
\hline & $(2,4,1,3),(3,1,4,2)$ & & \\
\hline & $(2,3,4,1),(4,1,2,3)$ & 6 & $1-2 t+2 t^{2}-t^{3}$ \\
\hline & $(3,4,1,2),(2,4,3,1)$, & & \\
\hline & $(3,2,4,1),(4,1,3,2)\}$, & 4 & $1-2 t+t^{2}$ \\
\hline & $(4,2,1,3)$ & & \\
\hline & $(4,2,3,1),(3,4,2,1)$, & 2 & $1-t$ \\
\hline & $(4,3,1,2)$ & & \\
\hline & $(4,3,2,1)$ & 1 & 1 \\
\hline
\end{tabular}

Moreover, $\chi_{(1,2,3,4,5)}=52, \chi_{(1,2,3,4,5,6)}=104$.

The natural conjecture that for $n$ fixed the maximal value of $\chi_{\sigma}$ is achieved at $\sigma=(1,2, \ldots, n)$ fails. Indeed, already for $n=5$ one has $\chi_{(1,3,2,4,5)}=\chi_{(1,2,4,3,5)}=$ $56, \chi_{(1,3,4,2,5)}=60$ (the latter value is maximal for $\mathbf{S}_{5}$ ).

It would be interesting to study the topology of $A_{\sigma}$. For $n=1,2,3$ in the real case all $A_{\sigma}$ are disconnected unions of cells. The same is apparently true for $n=4$ thus adding one more number - 52- to the list of the numbers of connected components of the set of all flags transversal to a given transversal pair of flags (see [A]). For $n>4$ the topology of $A_{\sigma}$ can be nontrivial.

Note that to each permutation $\sigma$ from $\mathbf{S}_{n}$ one can assign the permutation $\hat{\sigma}=$ $(\sigma, n+1)$ from $\mathbf{S}_{n+1}$. Evidently, $A_{\hat{\sigma}}$ is homotopically equivalent to $A_{\sigma}$ (in fact $A_{\hat{\sigma}}$ is 
a cylinder over $A_{\sigma}$ ). Hence, on the set of all possible permutations one obtains the generalized Bruhat ordering, whose maximal element is "the inverse permutation of all positive integers". Does cohomology of the corresponding $A_{\sigma}$ stabilize? If so, find the stable cohomology ring.

It would be also interesting to find the cohomology and the mixed Hodge structure of $A_{\sigma}$ in the complex case. We managed to obtain the answer for $n \leq 4$. In all this cases the corresponding mixed Hodge structure is pure, namely, $h_{i}^{i i}\left(A_{\sigma}\right)=\left|\chi_{\sigma}^{i i}\right|$ while all the other $h_{k}^{i j}$ vanish. It is tempting to prove that the same is true for all $A_{\sigma}$, but most likely it is a low dimensional effect. It is apparently easy to prove (decomposing $A_{\sigma}$ in the disjoint union of quasiprojective manifolds each diffeomorphic to the product of the certain numbers of copies of $\mathbf{C}$ 's and $\mathbf{C}^{*}$ 's) that in the mixed Hodge structure of $A_{\sigma}$ the $h_{k}^{i j}$ always vanish for $i \neq j$. On the other hand, the analogs of $A_{\sigma}$ for Grassmann manifolds turn out to be isomorphic to $\mathbf{G L}_{n}(\mathbf{C})$ while the mixed Hodge structure of these fails to be pure.

\section{REFERENCES}

[A] V. I. Arnold, Ten problems, The theory of singularities and its applications (series Advances in Soviet Mathematics) (to appear), AMS publications, Providence RI.

[D] A. H. Durfee, Algebraic varieties which are a disjoint union of subvarieties, Geometry \& Topology. Manifolds, Varieties \& Knots (C. McCrory and T. Schifrin, eds.), Marcel Dekker, New-York \& Boston, 1987, pp. 99-102.

[FF] A. T. Fomenko and D. B. Fuchs, A Course in Homotopical Topology, Nauka, Moscow, 1989.

[G] V. A. Ginzburg, Equivariant cohomology and Kaehlerian geometry, Funct. Anal. i ego Prilozh. 21 (1987), no. 4, 19-34.

[M] W. S. Massey, Homology and Cohomology Theory, Marcel Dekker, New-York \& Boston, 1978.

[V] O. Y. Viro, On integral calculus based on Euler characteristics, Topology \& GeometryRohlin Seminar (O. Y. Viro, ed.). Lecture Notes in Mathematics, vol.1346, SpringerVerlag, N.Y., 1988, pp. ??-??. 